\section{Evolución de una vasculitis renal asociada a anticuerpos anti-citoplasma de neutrófilos (ANCA) durante un embarazo. Caso clínico}

\author{
JORGE VEGA ${ }^{1,2,3}$, GONZALO P. MÉNDEZ ${ }^{4}$
}

\section{Antineutrophil cytoplasmic antibody (ANCA) associated renal vasculitis and pregnancy. Report of one case}

ANCA mediated vasculitis mainly occur between the fourth and fifth decade of life; therefore, it is very uncommon to see pregnant patients with the disease. Vasculitis may affect significantly the course of pregnancy; in turn pregnancy can change the course of vasculitis. We report a 20 years old woman with AN$C A$-mediated renal vasculitis lasting 10 years who consulted with a pregnancy of 15 weeks. She was in remission and had amenorrhea attributed to ovarian toxicity due to cyclophosphamide. Pregnancy had an uneventful course with spontaneous delivery at the $37^{\text {th }}$ week, giving birth to a healthy newborn. Proteinuria increased during the course of pregnancy with a mild deterioration of kidney function. During the year after delivery, she had nephrotic proteinuria and a worsening of renal function.

(Rev Med Chile 2016; 144: 1351-1355).

Key words: Anti-Neutrophil Cytoplasmic Antibody-Associated Vasculitis; Cyclophosphamide; Glomerulonephritis; Kidney Diseases; Pregnancy Complications; Proteinuria; Vasculitis.
'Departamento de Medicina,

Escuela de Medicina, Universidad de Valparaíso, Valparaíso, Chile. ${ }^{2}$ Sección Nefrología, Servicio de Medicina, Hospital Dr. Gustavo Fricke, Viña del Mar, Chile. ${ }^{3}$ Sección Nefrología, Servicio de Medicina, Hospital Naval A. Nef, Viña del Mar, Chile.

${ }^{4}$ Departamento de Anatomía Patológica, Facultad de Medicina, Pontificia Universidad Católica de Chile, Santiago, Chile.

Recibido el 10 de mayo de 2016, aceptado el 1 de agosto de 2016

Correspondencia a:

Dr. Jorge Vega

5 Norte 1035, Viña del Mar,

Chile.

Teléfono: 56-32-2974237

jvegastieb@gmail.com

\section{L} as vasculitis asociadas a anticuerpos anti-citoplasma de los neutrófilos (ANCA) habitualmente se presentan en mujeres en la cuarta o quinta década de la vida, por lo que es raro observar embarazos ${ }^{1,2}$. En las más jóvenes el uso de ciclofosfamida frecuentemente produce infertilidad ${ }^{3}$. Es por ello que la información disponible sobre embarazos y vasculitis asociadas a ANCA (VA-ANCA) es escasa.

Durante el embarazo, el aumento de glucocorticoides, progesterona y estrógenos induce cambios en linfocitos B, $\mathrm{T}$ y monocitos ${ }^{4,5}$. Como consecuencia, las enfermedades inmunomediadas se modifican, mejorando o empeorando durante el embarazo ${ }^{5}$. Los efectos del embarazo sobre las VA-ANCA y de éstas sobre el embarazo se co- nocen sólo por casos aislados o pequeñas series clínicas $^{2,6-12}$.

Comunicamos este inusual caso clínico con el objetivo de describir la evolución de la enfermedad en relación al embarazo, en una paciente con vasculitis renal mediada por ANCA.

\section{Caso clínico}

Mujer de 20 años con antecedentes de un parto a los 12 años de edad. Consultó por fatigabilidad y edema de tobillos. Existía anemia (hemoglobina: 9,5 g/dl), VHS 27: $\mathrm{mm} / \mathrm{h}$, uremia: $40 \mathrm{mg} / \mathrm{dl}$, creatinina: $1,28 \mathrm{mg} / \mathrm{dl}$, colesterol: 241 mg/dl y triglicéridos: $87 \mathrm{mg} / \mathrm{dl}$. La orina mostró 
proteinuria (+), 3-5 leucocitos y 10-12 eritrocitos por campo. TSH: > $100 \mathrm{uUI} / \mathrm{ml}$, T3 y T4 bajos y anticuerpos anti-tiroideos positivos. En atención primaria se interpretó sus síntomas como secundarios a anemia e hipotiroidismo y se prescribió hierro y tiroxina.

Un mes más tarde la fatigabilidad persistía a pesar de la normalización de TSH, no habían aparecido otros síntomas pero la anemia se había acentuado. La VHS fue $106 \mathrm{~mm} / \mathrm{h}$, creatinina: 5,2 $\mathrm{mg} / \mathrm{dl}$, clearance de creatinina $(\mathrm{ClCr}): 17,0 \mathrm{ml} /$ $\mathrm{min} / 1,73 \mathrm{~m}^{2}$, urea: $109 \mathrm{mg} / \mathrm{dl}$, proteinuria: 1.700 $\mathrm{mg} /$ día, persistiendo la hematuria microscópica. Presión arterial 110/60 mmHg. Fue hospitalizada. Al examen físico no había otros signos fuera de la palidez y edema de tobillos. La radiografía de tórax, senos paranasales y ecotomografía abdominal fueron normales, no encontrándose alteraciones renales. Se sospechó una glomerulonefritis rápidamente progresiva (GNRP). Los anticuerpos antinucleares y anti-DNA, cuantificación de inmunoglobulinas, factor reumatoideo, C’3 y C'4 fueron negativos o normales. Los ANCA (IFI) fueron positivos $1 / 640$ con patrón perinuclear (no se efectuó Elisa para anticuerpos anti-MPO ni anti-PR3). Se administró metilprednisolona $1 \mathrm{~g} /$ por 3 veces y ciclofosfamida $500 \mathrm{mg}$ por vía endovenosa, continuando con prednisona $60 \mathrm{mg} /$ día y ciclofosfamida $100 \mathrm{mg} /$ día oral. Se realizó una biopsia renal, la cual mostró por examen de microscopía óptica 29 glomérulos, 23 de los cuales estaban obsoletos, existiendo compromiso por crecientes fibrocelulares en 17\% y fibrosas en $52 \%$ de los glomérulos (Figura 1). Tres glomérulos mostraban crecientes de predominio celular $(10 \%)$. La zona túbulo intersticial presentaba atrofia tubular moderada (33\%) e infiltrado inflamatorio linfo-plasmocitario multifocal. Los vasos eran de estructura normal. El tejido para inmunofluorescencia contuvo 3 glomérulos, los cuales tenían fibrina conformando imágenes de crecientes. No hubo reactividad glomerular para C3, C1q, IgA ni IgG. El glomérulo examinado por microscopía electrónica conservaba su arquitectura; no se encontraron depósitos electrón-densos en él y sólo mostraba desaparición pedicelar extensa. Se diagnosticó glomerulonefritis crecéntica crónica y activa de carácter "pauci inmune".

Tres meses más tarde, el estado general y función renal de la paciente habían mejorado (creatinina: 2,0 mg/dl; proteinuria: $\mathrm{m} 1.270 \mathrm{mg} / 24 \mathrm{~h}$ ) recibiendo prednisona, ciclofosfamida, enalapril, tiroxina y lovastatina. Al año, creatinina: $1,6 \mathrm{mg} /$ dl; proteinuria: $1.290 \mathrm{mg} / 24 \mathrm{~h}$. Dos años después refirió amenorrea, bochornos y sudoración; sospechándose toxicidad por ciclofosfamida, lo que fue confirmado por bajos niveles de estrógenos y elevación de gonadotrofinas. Se cambió la ciclofosfamida por azatioprina $50 \mathrm{mg} /$ día. Las menstruaciones no reaparecieron. Durante este

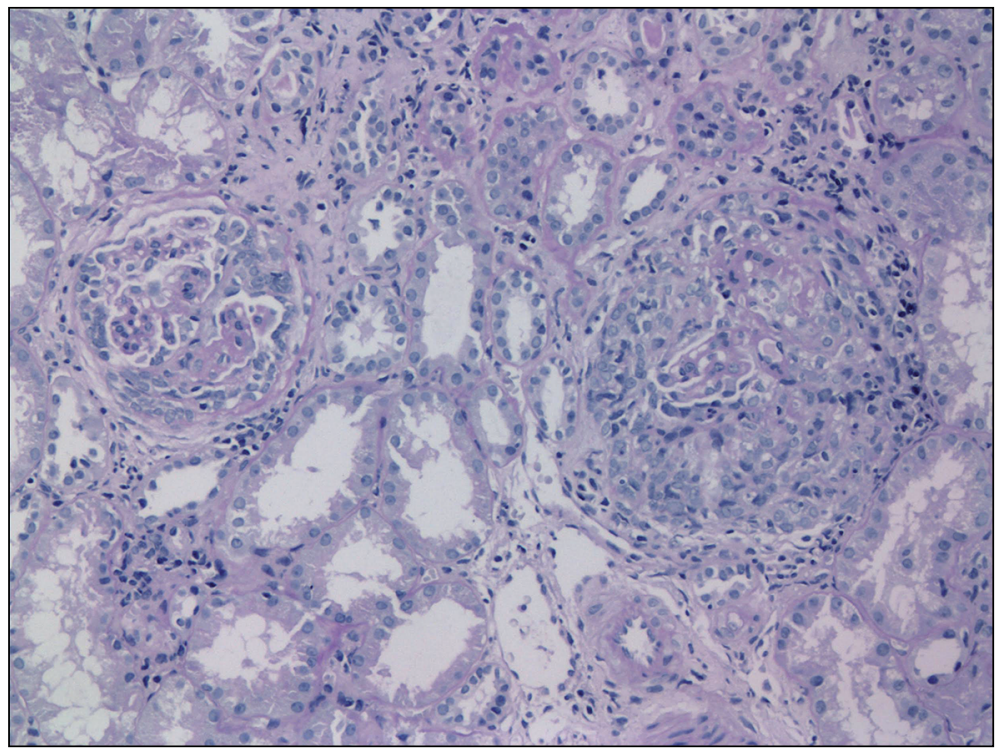

Figura 1. Microscopia óptica de la biopsia renal. Se reconoce un área de corteza con dos glomérulos, ambos comprometidos en prácticamente toda su circunferencia por lesiones crecénticas celulares. El intersticio periglomerular de ambos muestra focos con infiltrado linfocitario moderado (PAS, 200x). 
período no aparecieron manifestaciones clínicas de compromiso de otros órganos o sistemas, fuera de los mencionados.

Diez años después del diagnóstico presentó sangrado genital. La ecotomografía pelviana sorpresivamente mostró un embarazo de 15 semanas. El enalapril se suspendió manteniendo azatioprina y prednisona. La presión arterial era normal, no había hematuria, ClCr: $58 \mathrm{ml} /$ $\mathrm{min} / 1,73 \mathrm{~m}^{2}$, creatinina: $1,5 \mathrm{mg} / \mathrm{dl}$ y la proteinuria había aumentado a $2.960 \mathrm{mg} / 24 \mathrm{~h}$. El embarazo transcurrió con normotensión y sin edema, la proteinuria aumentó hasta $4.440 \mathrm{mg} / 24 \mathrm{~h}$ a las 33 semanas, asociada a una reducción del $\mathrm{ClCr}$ a $50,9 \mathrm{ml} / \mathrm{min} / 1,73 \mathrm{~m}^{2}$ con ascenso de creatinina a $1,7 \mathrm{mg} / \mathrm{dl})$. Un parto espontáneo ocurrió a las 37 semanas, sin complicaciones. El recién nacido pesó 2.440 g y no presentó malformaciones u otras patologías evidentes.

Dos semanas después, la proteinuria aumentó a $11.870 \mathrm{mg} / 24 \mathrm{~h}, \mathrm{ClCr}: 50 \mathrm{ml} / \mathrm{min} / 1,73 \mathrm{~m}^{2}$ y no había hematuria. Se indicó suspender la lactancia para reiniciar la terapia anti-proteinúrica pero la paciente no lo aceptó, abandonando los controles. Reapareció a los 7 meses del parto dando lactancia. La proteinuria fue $12.700 \mathrm{mg} / 24 \mathrm{~h}, \mathrm{ClCr}: 46,6 \mathrm{ml} /$ $\mathrm{min} / 1,73 \mathrm{~m}^{2}$ y creatinina: $2,0 \mathrm{mg} / \mathrm{dl}$. Se indicó enalapril $20 \mathrm{mg} \mathrm{c} / 12 \mathrm{~h}$ y azatioprina $100 \mathrm{mg} /$ día. $\mathrm{Al}$ año del parto la situación había empeorado, la uremia: $94 \mathrm{mg} / \mathrm{dl}$, creatinina: $3,1 \mathrm{mg} / \mathrm{dl}, \mathrm{ClCr}$ : $41 \mathrm{ml} / \mathrm{min} / 1,73 \mathrm{~m}^{2}$ y proteinuria: $4.850 \mathrm{mg} / 24 \mathrm{~h}$. La determinación de ANCA por IF fue positiva a 1/640 y la determinación por ELISA mostró especificidad de los anticuerpos por mieloperoxidasa. Se cambió la azatioprina por ciclofosfamida y se aumentó la dosis de corticoides. Ello, en los 3 meses siguientes, estabilizó la velocidad de filtración glomerular $\left(45 \mathrm{ml} / \mathrm{min} / 1,73 \mathrm{~m}^{2}\right)$ y redujo la proteinuria a rangos sub-nefróticos.

\section{Discusión}

Esta paciente inició su enfermedad a los 20 años sospechándose una GNRP, que fue confirmada con biopsia renal que mostró una GN crecéntica pauci-inmune con un severo daño glomerular, asociada a anticuerpos del tipo P-ANCA. Se prescribió ciclofosfamida y corticoides, mejorando rápidamente la función renal, manteniéndose durante 22 meses, en que se cambió ciclofosfamida por azatioprina por toxicidad ovárica. Este cambio no produjo reaparición de las menstruaciones por lo que se asumió un daño ovárico definitivo.

Transcurridos 10 años de su enfermedad y estando en remisión, se diagnosticó un embarazo de 15 semanas. Este evolucionó sin complicaciones obstétricas ni empeoramiento en la función renal hasta las 33 semanas, en que se produjo una reducción en la función renal e incremento en la proteinuria. La paciente tuvo un parto espontáneo a las 37 semanas obteniéndose un recién nacido sano.

Las variedades de VA-ANCA de acuerdo a la clasificación de Chapel Hill 2012 son: poliangeitis microscópica, granulomatosis con poliangeitis (Wegener), granulomatosis eosinofílica con poliangeitis (Churg-Strauss) y vasculitis limitadas a un solo órgano (Ej: riñón $)^{14}$. Esta paciente, al no presentar vasculitis de otros órganos puede ser clasificada en la última variedad.

Las VA-ANCA diagnosticadas con anterioridad a un embarazo y que han permanecido en remisión durante años, como en esta paciente, pueden reactivarse durante la gestación produciendo efectos en la madre y en el feto. También el embarazo puede modificar la evolución de la vasculitis con deterioro importante de órganos como riñón ${ }^{2}$.

La mayoría de las publicaciones disponibles corresponden a pacientes con granulomatosis con poliangeitis (GPA) $)^{2,6,8,10-12,14}$. Un 25\% de las pacientes con GPA recaen en el embarazo en el $2^{\circ}$ y $3^{\text {er }}$ trimestre o en el post-parto inmediato ${ }^{1,15}$. Las que conciben estando activa la enfermedad experimentan un empeoramiento de los síntomas. También se han comunicado recaídas después de un aborto ${ }^{15}$. La GPA tiene un efecto adverso en el embarazo cuando la enfermedad está activa o aparece por primera vez. Hay una mayor tasa de abortos y de mortalidad materna. La pre-eclampsia, prematuridad, restricción de crecimiento intrauterino, cesáreas, desprendimientos placentarios, placenta previa y pérdidas fetales tardías son más frecuentes ${ }^{3}$. Las enfermas con GPA tienen mayor riesgo de trombosis y estenosis sub-glótica, originando problemas de intubación durante la anestesia $^{1,2}$. Se ha detectado el paso transplacentario de anticuerpos anti-PR3 al feto, pero sin experimentar vasculitis9.

Hay pocas publicaciones de embarazo y granulomatosis eosinofílica con poliangeitis (EGPA) ${ }^{15,16}$. La mitad de las pacientes recaen 
presentando empeoramiento del asma, mononeuritis múltiple, agravación de la cardiopatía incluso con falla cardiaca y muerte. Cuando la enfermedad permanece en remisión nacen niños sanos pero de bajo peso. Cuando la enfermedad debuta durante un embarazo hay una alta tasa de muerte materna y fetal ${ }^{2}$.

Los casos comunicados de poliangeitis microscópica (MPA) y embarazo son escasos. Hay reportes de embarazos exitosos ${ }^{15} y$ con hemorragia pulmonar ${ }^{17}$. La transmisión transplacentaria de anticuerpos anti-MPO al feto puede originar un síndrome riñón-pulmón después del nacimiento $^{2,18,19}$.

Existe menos información de embarazos y VA-ANCA limitadas a un órgano ${ }^{6,7}$. En una mujer con compromiso renal, anticuerpos P-ANCA y anti-MPO, se observó incremento de la proteinuria durante el embarazo con empeoramiento de la función renal, terminando en parto prematuro con un niño sano ${ }^{6}$. En esta enferma también se observó un aumento de la proteinuria durante el embarazo, sin embargo, la función renal sólo se redujo levemente antes del parto.

En las reactivaciones de las VA-ANCA durante un embarazo o en las de novo, se ha recomendado el uso de corticoides y azatioprina. Metotrexato, micofenolato y ciclofosfamida son drogas teratogénicas ${ }^{1,2}$. Ciclofosfamida se ha utilizado en el tercer trimestre sin complicaciones fetales ${ }^{2}$. Se han usado también ciclosporina, inmunoglobulina endovenosa, plasmaféresis y rituximab $b^{1,7,14,20,21}$.

Con la información actualmente disponible se han hecho las siguientes recomendaciones:

a) Antes de iniciar un embarazo debe obtenerse una remisión prolongada ${ }^{15}$.

b) Debe hacerse un cambio a drogas no teratogénicas como azatioprina ${ }^{2}$.

c) En una paciente que ha tenido una reactivación en un embarazo previo no debe asumirse que esto se repetirá en un embarazo siguiente ${ }^{15}$.

d) Durante el embarazo debe monitorizarse frecuentemente la función renal, serología de autoinmunidad, parámetros hematológicos y de inflamación ${ }^{2}$.

e) En mujeres en remisión con azatioprina y corticoides es recomendable mantener dicha terapia durante el embarazo ${ }^{1}$.

f) La aspirina en dosis bajas puede utilizarse para prevenir pre-eclampsia, especialmente en enfermas con hipertensión o daño renal ${ }^{3}$. g) Las recaídas deben ser tratadas agresivamente, idealmente con drogas seguras durante el embarazo ${ }^{2}$.

h) Después del parto el estado de inmuno-tolerancia desaparece y ocurre la reconstitución inmune, por lo que las pacientes deben ser controladas por la posibilidad de reactivación de la enfermedad ${ }^{14}$.

i) El riesgo de muerte de la madre debe balancearse con el riesgo potencial para el feto y las decisiones deben ser multidisciplinarias considerando la opinión de la paciente ${ }^{2}$.

\section{Referencias}

1. Grygiel-Górniak B, Puszczewicz M. Granulomatosis with polyangiitis in pregnancy - clinical implications and treatment posibilities. Eur Rev Med Pharmacol 2015; 9: 2331-5.

2. Pagnoux C, Le Guern V, Goffinet F, Diot E, Limal N, Pannier E, et al. Pregnancies in systemic necrotizing vasculitides: report on 12 women and their 20 pregnancies. Rheumatology (Oxford) 2011; 50: 953-61.

3. Sangle SR, Vounotrypidis P, Briley A, Nel L, Lutalo PM, Sánchez-Fernández S, et al. Pregnancy outcome in patients with systemic vasculitis: a single-centre matched case-control study. Reumatology (Oxford) 2015; 54: 1582-6.

4. Cutolo M, Capellino S, Straub RH. Oestrogens in rheumatic diseases: friend or foe? Rheumatology (Oxford) 2008; 47 Suppl 3: iii2-5.

5. Ostensen M, Brucato A, Carp H, Chambers C, Dolhain RJ, Doria A, et al. Pregnancy and reproduction in autoimmune rheumatic diseases. Rheumatology (Oxford) 2011; 50: 657-64.

6. Oshima M, Kitajima S, Toyama T, Hara A, Kitagawa $\mathrm{K}$, Iwata $\mathrm{Y}$, et al. Successful delivery in a patient with antineutrophil cytoplasmic antibody-associated glomerulonephritis. Intern Med 2013; 52: 1605-9.

7. Presta P, Presta P, Fuiano G, Lombardi G, Simeoni M, Rivoli L, et al. Successful conception and pregnancy in p-ANCA-associated vasculitis in course of treatment with immunosuppressive drugs and renal replacement therapy. Int J Rheum Dis 2015; 18: 470-2.

8. Kayatas S, Asoglu MR, Selcuk S, Sargin MA. Pregnancy in a patient with Wegener's granulomatosis: a case report. Bull NYU Hosp Jt Dis 2012; 70: 127-9.

9. Alfhaily F, Watts R, Leather A. Wegener's granulomatosis occurring de novo during pregnancy. Clin Exp Rheumatol 2009; 27 (1 Suppl 52): S86-88.

10. Sahni V, Agarwal SK, Singh NP, Sikdar S, Yadav A, 
Wadhwa A, et al. Successful pregnancy in untreated limited Wegener's granulomatosis. Med J Malaysia 2005; 60: 492-4

11. Auzary C, Huong DT, Wechsler B, Vauthier-Brouzes D, Piette JC. Pregnancy in patients with Wegener's granulomatosis: report of five cases in three women. Ann Rheum Dis 2000; 59: 800-4.

12. Bessias N, Moulakakis KG, Lioupis C, Bakogiannis $\mathrm{K}$, Sfyroeras G, Kakaletri K, et al. Wegener's granulomatosis presenting during pregnancy with acute limb ischemia. J Vasc Surg 2005; 42: 800-4.

13. Jennette JC, Falk RJ, Bacon PA, Basu N, Cid MC, Ferrario F, et al. 2012 revised International Chapel Hill Consensus Conference Nomenclature of Vasculitides. Arthritis Rheum 2013; 65: 1-11.

14. Soh MC, Nelson-Piercy C. High-risk pregnancy and the rheumatologist. Rheumatology (Oxford) 2015; 54: 57287.

15. Seo P. Pregnancy and vasculitis. Rheum Dis Clin N Am 2007; 33: 299-317.
16. Connolly JO, Lanham JG, Partridge MR. Fulminant pregnancy-related Churg-Strauss syndrome. Br J Rheumatol 1994; 33: 776-7.

17. Savage C, Winearls C, Evans D, Rees AJ, Lockwood CM. Microscopic polyarteritis: presentation, pathology, and prognosis. QJM 1985; 56: 467-83.

18. Schlieben DJ, Korbet SM, Kimura RE, Schwartz MM, Lewis EJ. Pulmonary-renal syndrome in a newborn with placental transmission of ANCAs. Am J Kidney Dis 2005; 45: 758-61.

19. Bansal PJ, Tobin MC. Neonatal microscopic polyangiitis secondary to transfer of maternal myeloperoxidase-antineutrophil cytoplasmic antibody resulting in neonatal pulmonary hemorrhage and renal involvement. Ann Allergy Asthma Immunol 2004; 93: 398-401.

20. Masterson R, Pellicano R, Bleasel K, McMahon LP. Wegener's granulomatosis in pregnancy: a novel approach to management. Am J Kidney Dis 2004; 44: e68-72.

21. Jayne D. Rituximab treatment of vasculitis. Clin J Am Soc Nephrol 2010; 5: 1359-62. 